\title{
Web PCR
}

\section{Stacey McCann}

Every year scientists introduce new polymerase chain reaction (PCR) modifications: multiplex PCR, hemi-nested PCR, anchorligated PCR, etc. In this spirit, I now introduce-Web PCR. Unlike other PCR modifications that involve experimental protocols, Web PCR is about making the first steps of a PCR experiment as simple as pointing and clicking in your browser. By using resources available on the Internet, you can design, test, and order PCR primers, as well as optimize PCR conditions.

\section{Primer databases and testing}

Molecular biologists increasingly use the web to build databases through the voluntary submission of data. Scientists pool their data to form sequence databases such as GenBank, EMBL, and DDBJ, and anyone can search them, greatly reducing both research time in the library and the amount of experimental duplication. The PCR primers database maintained by the European Bioinformatics Institute (http://www.ebi.ac.uk/dbases/ primers/primers_home.html) contains previously tested primers, so researchers can increase their chances for success by using PCR primers that have already proved successful. Primer searches can be done by target, sequence, species, and contributing author.

When previously tested primers are not available for a particular experiment, scientists often use primer design and analysis programs to find appropriate primers. The latest primer programs represent the shift from stand-alone computer applications to webbased applications. The first PCR programs available on the Internet were simple forms, in which the user could enter data into fields and then submit the form. The server would process and analyze the data, and return the results. Newer applications use Internet programming languages such as Java to allow completely interactive applications to run directly in the browser. As the use of Internet languages expands, more sophisticated programs are available to allow primer design and analysis without ever leaving the browser.

For example, NetPrimer analyzes primers for potentially detrimental characteristics directly within the browser. Primers can fail for a number of reasons, including formation of hairpins and dimers within a primer or

Stacey McCann is in the cancer biology program at Stanford University School of Medicine(smccann@leland.stanford.edu). cross-dimers between two primers. NetPrimer will print a primer analysis report that outlines potential secondary structures for that primer pair. Also, even though primers obtained from a database have been used before, users should check them for sec-

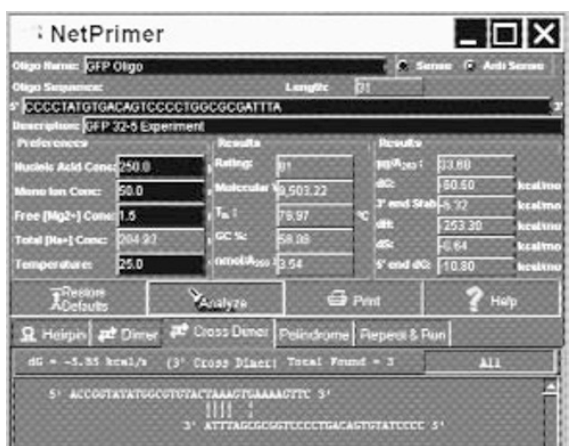

ondary structures to increase their chance of success. Scientists can find the NetPrimer page maintained by Premier Biosoft International (Palo Alto, CA) at http://www.premier biosoft.com/netprimer.html.

\section{Ordering primers}

In keeping with the trend toward e-commerce, most oligonucleotide synthesis companies now offer web ordering. In fact, some report more than $50 \%$ of sales coming from the web. The system is convenient for both buyers and sellers: Rather than taking the trouble to print or hand-copy primer sequences and then faxing them to the company, scientists find it much easier to paste primer sequences directly into a web form, enter a credit card or purchase order number, and directly order the primers. And companies return fewer orders because of missing or incorrect information.

Internet order forms often contain more information about custom services, such as oligonucleotide end modifications. Some companies also offer primer analysis programs directly from their order pages, reducing the frequency of customer complaints by reducing the probability of ordering nonfunctional primers. Purchasers can track orders throughout the synthesis and shipping processes. For example, Genosys (The Woodlands, TX), a high-throughput oligosynthesis company, offers web ordering at http://www.genosys.com/order01.html.

\section{Protocols and calculations}

Protocol jump stations allow scientists to find protocols for an experiment without leaving their labs. Jump sites (web sites that provide well-organized links to other pages on specific topics but offer no content of their own) provide a convenient way of locating sites containing specific information. They can also include ratings of the links and search capability. Scientific protocols and methods are particularly well suited for jump station formats, which organize these protocols by topic for easy access. At http://biology.neehow.org/ wonderful/protocols/pcrtech.html, searchers can obtain PCR protocols as well as protocols for other molecular biology applications.

The web also offers calculators for a variety of scientific applications. Available calculators range from Metric/English system conversions to quadratic equation solutionsconvenient for scientists who do not do these calculations frequently enough to warrant a stand-alone program. Web-based calculators are available to facilitate PCR experiments as well. NetPrimer can be used to calculate primer concentration and optimal annealing temperature.

\section{Troubleshooting}

The Internet provides a forum by which scientists can easily gain access to other scientists' experimental experience. Those new to PCR can find a wealth of information on getting started, designing primers, and optimizing PCR, and they can overcome obstacles by drawing on the experience of others in addressing common problems.

Trouble-shooting guides address many common problems. What if the amplification fails? A PCR troubleshooting guide such as http://medgen.iupui.edu/ ohenega/Trblesht.ht $\mathrm{ml}$ provides solutions for a wide variety of problems. Many thermocycler and oligonucleotide synthesis companies also provide troubleshooting guides. For thornier problems, scientists can post a message on one of the biology usenet groups such as bionet.molbio.methds-reagnts to get advice from helpful colleagues.

The Internet provides many options for increasing the efficiency of scientific research and reducing the number of failed experiments, including PCR. The rapid spread of information allows researchers to learn from the experience of other scientists (e.g., use of primer databases and troubleshooting guides), as well as to find appropriate computer programs to analyze primer characteristics for optimal amplification. Increasingly, scientists will look to the web before starting any new experiment. 